\title{
Fractionation and Crystallization of Isotactic Polypropylenes Prepared Using Homogenous Metallocene Catalyst
}

\author{
Ismael AMER ${ }^{1}$ *, Touhami MOKRAN ${ }^{l}$ \& Albert VAN REENEN ${ }^{2}$ \\ ${ }^{1}$ Department of Civil and Chemical Engineering, College of Science, Engineering and Technology, \\ University of South Africa, Private Bag X6, Florida 1710, Johannesburg, SOUTH AFRICA \\ ${ }^{2}$ Department of Chemistry and Polymer Science, University of Stellenbosch, South Africa, Private \\ Bag X1, Matieland, 7602, SOUTH AFRICA. \\ *Corresponding author: ismaelamer77@ yahoo.co.za, Tel: +27116709382
}

Received: 13 August 2015 Revised: 25 August 2015 Accepted: 25 August 2015 Online: 7 September 2015

\section{Keywords:}

Characterization; Metallocene Catalyst; Polymerization; Polypropylene

\begin{abstract}
A b s tract
A series of polypropylenes were prepared using rac-ethylene-bis(4,5,6,7tetrahydro-1-indenyl) zirconium dichloride $\left(\mathrm{Et}(\mathrm{H} 4 \mathrm{Ind})_{2} \mathrm{ZrCl}_{2}(\mathrm{EI}(4 \mathrm{H}))\right.$ as a homogeneous metallocene catalyst. The molecular weight of the polypropylene polymers was controlled using molecular hydrogen which was used as a transfer agent. To obtain materials with differing molecular weight and tacticities, polymers were fractionated with prep-TREF. Polypropylene polymers and fractions were fully characterized using various analytical techniques: differential scanning calorimetry (DSC), wide-angle X-ray diffraction (WAXD), ${ }^{13} \mathrm{C}$ nuclear magnetic resonance spectroscopy $\left({ }^{13} \mathrm{C}-\mathrm{NMR}\right)$ and high temperature gel permeation chromatography (HT-GPC). It was found that the addition of molecular hydrogen to propylene polymerizations catalyzed using $\mathrm{EI}(4 \mathrm{H})$ catalyst system resulted in a reduction in polymer molecular weight and isotacticity. Furthermore, the molecular weight, the isotacticity and the degree of crystallinity of the TREF fractions increased significantly with increasing elution temperature for all samples. Also, only the $\alpha$ crystal form existed in all TREF fractions of different samples.
\end{abstract}

\section{Introduction}

Metallocene catalyst systems have been around for just as long as the Ziegler-Natta systems (Auriemma et al., 2001). Metallocenes are special members of a class of organometallic compounds. They are activated with methylaluminoxane (MAO) to form single-site catalyst systems that are effective for $\alpha$-olefin polymerization. These catalysts produce polymers with uniform and controlled properties. Chiral metallocene catalysts possessing $C 2$-symmetry have been widely studied in order to optimize, and to better understand, their ability to catalyze the stereoregular polymerization of propylene (Busico et al., 1998; Soga \& Shiono, 1997). Ansa C2-symmetric metallocene catalysts are highly isoselective in propylene polymerization and have high activity. This allows for their use in most industrial applications for the production of polypropylene homopolymers and polypropylene copolymers. Control of the molecular weight of polymers produced by $\mathrm{C} 2$-metallocene catalysts can be easily achieved by introducing hydrogen to the polymerization system or by increasing the reaction temperature. Unlike conventional Ziegler-Natta catalysts, metallocenes require only small amounts of hydrogen to significantly reduce the molecular weight of polymer produced (Lin et al., 2000). 
This study reports on propylene polymerization and analysis of the microstructure, molecular weight, molecular weight distribution and thermal properties of polymers produced using racethylene-bis(4,5,6,7-tetrahydro-1-indenyl) zirconium dichloride, $\mathrm{Et}(\mathrm{H} 4 \mathrm{Ind})_{2} \mathrm{ZrCl}_{2}(\mathrm{EI}(4 \mathrm{H})$ ) (Figure 1) as metallocene catalyst in the absence and presence of molecular hydrogen as transfer agent. In order to establish a correlation between the molecular weight and the crystallization of these polymers, fractionation of the materials according to their crystallizability was performed by means of TREF.

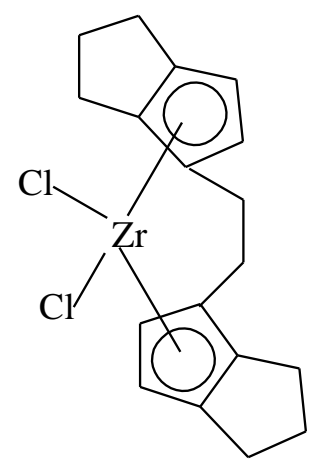

Figure 1: $r a c-E t(H 4 I n d){ }_{2} \mathrm{ZrCl}_{2}(\mathrm{EI}(4 \mathrm{H}))$.

\section{Methodology}

\section{Material}

Propylene was obtained from Sigma-Aldrich and used as received. High purity argon and hydrogen were obtained from Afrox (South Africa). Toluene (Sigma-Aldrich) was dried by refluxing over sodium/benzophenone and then distilling under an inert gas atmosphere. MAO was purchased from Sigma-Aldrich (10 wt \% solution in toluene) and used as received. The EI $(4 \mathrm{H})$ catalyst used in this study was obtained from Sigma-Aldrich and used as received. Metallocene catalysts comprising group 4 metallocene complexes and MAO are air and moisture sensitive, and thus experiments must be performed under an inert atmosphere and in dry solvents.

\section{Polymerization Procedure}

All reactions were carried out under an inert gas atmosphere using standard Schlenk techniques. The polymerization reactions were carried out in a $350-\mathrm{mL}$ stainless steel Parr autoclave with an inlet and a pressure gauge. Typically, the reactor was charged with MAO (10 wt \% solution in toluene), catalyst $(0.55 \mu \mathrm{mol}$ in $5 \mathrm{~mL}$ toluene) and toluene $(30 \mathrm{~mL})$. The catalyst solution was stirred for 5 minutes and then the monomer was added. The reactor was pressurized with hydrogen and the content stirred for 1 hour at room temperature. The following polymerization conditions were used for all reactions: the catalyst/MAO ratio was kept at 1:2000, 4-5 g propylene was used, all reactions were carried out at room temperature, and different amounts of hydrogen were used as terminating agent to control the molecular weight. After 1 hour the reaction was quenched with $10 \% \mathrm{HCl} / \mathrm{MeOH}$. The 
resulting polymer was filtered off, washed several times with methanol and then dried under vacuum at $80{ }^{\circ} \mathrm{C}$ for 15 hours to yield about $2-4 \mathrm{~g}$ of polypropylene as a white powder.

\section{TREF Procedure}

Typically $3.0 \mathrm{~g}$ of polymer was introduced to a $1000 \mathrm{~mL}$ glass reactor containing $300 \mathrm{~mL}$ xylene stabilized with $0.06 \mathrm{wt} \%$ Irganox 1010 . The polymer was dissolved at $130{ }^{\circ} \mathrm{C}$ for about 90 minutes. The hot polymer solution was mixed with preheated washed sea sand (Aldrich, as an inert support). The glass reactor was cooled from $130{ }^{\circ} \mathrm{C}$ to $20{ }^{\circ} \mathrm{C}$ at a rate of $1.5{ }^{\circ} \mathrm{C} / \mathrm{h}$. The solution-sand mixture was loaded into a steel elution column. Fractions of the polymer, with increasing crystallinity, were then eluted with xylene at set (increasing) temperatures. Typically fractions were isolated after elution at 7 temperatures $\left(25,60,80,90,100,110\right.$ and $\left.130{ }^{\circ} \mathrm{C}\right)$. Subsequently the polymer fractions were recovered by removing the xylene under reduced pressure and drying at $50{ }^{\circ} \mathrm{C}$ (vacuum oven).

\section{Characterization Techniques}

Polypropylene polymers were characterized by HT-GPC (Polymer Labs PL-GPC 220, flow rate of 1 $\mathrm{mL} / \mathrm{min}$ in 1,2,4 trchlorobenzene as solvent stabilized with 2,6-di-tert-butyl-4-methylphenol at 140 ${ }^{\circ} \mathrm{C}$ ). DSC (TA instruments Q100 DSC, 2nd heating cycle at $10{ }^{\circ} \mathrm{C} / \mathrm{min}$ used for determination of $\mathrm{T}_{\mathrm{m}}$, $\mathrm{T}_{\mathrm{c}}$ determined from first cooling cycle). ${ }^{13} \mathrm{C}$ NMR spectra were recorded at $120{ }^{\circ} \mathrm{C}$ on a Varian Unity Inova NMR spectrometer equipped with an Oxford magnet (14.09 T), operating at $600 \mathrm{MHz}$, using a $5 \mathrm{~mm}$ inverse detection PFG probe. Samples $(60-80 \mathrm{mg})$ for ${ }^{13} \mathrm{C}$ NMR analyses were dissolved at 110 ${ }^{\circ} \mathrm{C}$ in a 9:1 mixture of TCB and benzene- $\mathrm{d}_{6}\left(\mathrm{Bz}_{6}\right)$. WAXD analyses were performed at iThemba LABS (South Africa) on a Bruker AXS D8 ADVANCE diffractometer at room temperature with filtered $\mathrm{CuK} \alpha$ radiation. All samples were scanned at $2 \theta$ angles, ranging from $6^{\circ}$ to $50^{\circ}$, with a sampling width of $0.02^{\circ}$, where $2 \alpha$ is the diffraction angle.

\section{Result and Discussion}

\section{Characterization of the Non-fractionated Polypropylenes}

The characteristics of the polypropylenes prepared using EI(4H) metallocene catalyst in the absence and presence of different amounts of molecular hydrogen are summarized in Table 1. The samples prepared using EI $(4 \mathrm{H})$ were coded as D9-D3 in Table 1. It is obvious that the use of hydrogen strongly affects polymer molecular weight. The presence of a small amount of hydrogen caused a remarkable reduction in polymer molecular weights $\left(M_{w}=24809 \mathrm{~g} \mathrm{~mol}^{-1}\right.$ in the absence of hydrogen deceased to $\mathrm{M}_{\mathrm{w}}=7188 \mathrm{~g} \mathrm{~mol}^{-1}$ in the presence of $0.3 \mathrm{wt} \%$ of hydrogen). This decrease in the molecular weight can be explained as a result of an increase of chain termination by hydrogen. 
Table 1: Results of propylene polymerization carried out with EI(4H) catalyst system using various concentrations of hydrogen at room temperature

\begin{tabular}{cccccccccc}
\hline Run & $\begin{array}{c}\mathrm{H}_{2} \\
(\mathrm{wt} \%)^{\mathrm{a}}\end{array}$ & Activity & $\mathrm{M}_{\mathrm{w}}{ }^{\mathrm{c}}$ & $\mathrm{PD}$ & mmmm $\%^{\mathrm{d}}$ & $\begin{array}{c}\mathrm{T}_{\mathrm{m}} \\
\left({ }^{\circ} \mathrm{C}\right)\end{array}$ & $\begin{array}{c}\mathrm{T}_{\mathrm{c}} \\
\left({ }^{\circ} \mathrm{C}\right)\end{array}$ & $\begin{array}{c}\Delta \mathrm{H}_{\mathrm{m}}{ }^{\mathrm{e}} \\
(\mathrm{J} / \mathrm{g})\end{array}$ & $\begin{array}{c}\mathrm{X}_{\mathrm{c}}{ }^{\mathrm{f}} \\
(\%)\end{array}$ \\
\hline D9 & 0.0 & 1785 & 24809 & 2.0 & 88.0 & 138.0 & 107.7 & 87.4 & 41.8 \\
D8 & 0.3 & 2171 & 7188 & 2.0 & 85.8 & 137.2 & 104.4 & 96.9 & 46.4 \\
D7 & 0.7 & 2191 & 5262 & 1.9 & 85.1 & 135.0 & 102.8 & 101.4 & 48.5 \\
D6 & 1.0 & 2235 & 4369 & 1.9 & 81.9 & 135.6 & 105.8 & 102.4 & 49.0 \\
D5 & 1.3 & 2287 & 3290 & 1.8 & 79.0 & 132.0 & 99.8 & 79.4 & 38.0 \\
D4 & 1.7 & 2151 & 2825 & 1.8 & 77.7 & 132.1 & 102.4 & 94.9 & 45.4 \\
D3 & 2.0 & 2424 & 2244 & 1.7 & 70.3 & 127.3 & 95.8 & 81.7 & 39.1 \\
\hline
\end{tabular}

${ }^{\mathrm{a}}\left(\mathrm{g} \mathrm{H}_{2} / \mathrm{g}\right.$ propylene $) \times 100,{ }^{\mathrm{b}}(\mathrm{kg} \mathrm{PP} /(\mathrm{mol} \mathrm{Zr} . \mathrm{h})),{ }^{\mathrm{c}}$ determined by GPC,${ }^{\mathrm{d}}$ determined by ${ }^{13} \mathrm{C} \mathrm{NMR},{ }^{\mathrm{e}}$ determined by DSC, ${ }^{\mathrm{f}} \Delta \mathrm{H}_{\mathrm{m}}=209 \mathrm{~J} / \mathrm{g}$ of PP $100 \%$ crystallinity (Deukkil et al., 2005)

The isotacticity (measured as $\mathrm{mmmm} \%$ ) is affected by hydrogen. Conflicting results have been reported for different systems. Tsutsui et al. (1990) reported that a slight decrease in stereoregularity, from 91.7 to $89.0 \% \mathrm{~mm}$ triads, for the $\mathrm{rac}-\mathrm{C}_{2} \mathrm{H}_{4}(1-\mathrm{Ind}){ }_{2} \mathrm{ZrCl}_{2} / \mathrm{MAO}$ catalyst system. A stronger negative effect was found for bis(2-arylindenyl)Zr/MAO catalysts by Lin and Waymouth (1999). No effect of hydrogen on isotacticity has been found in liquid propylene at $50{ }^{\circ} \mathrm{C}$ with the rac- $\mathrm{Me}_{2} \mathrm{C}(3-\mathrm{t}-\mathrm{Bu}-1-\mathrm{Ind})_{2}-\mathrm{ZrCl}_{2} / \mathrm{MAO}$ catalyst system (Moscardi et al., 1999). Carville et al. (1997) also reported that low hydrogen levels do not influence tacticity. In the present study, the isotacticity of polypropylenes produced by $\mathrm{EI}(4 \mathrm{H})$ catalyst is significantly decreased (from $88.0 \%$ in the absence of hydrogen to $70.3 \%$ in the presence of $2.0 \mathrm{wt} \%$ hydrogen), as can be seen in Figure 2 and Table 1 . The decrease in the isotacticity of these polymers is due to the increase of stereo-irregular pentads, [mmmr], $[\mathrm{mmrr}]$ and $[\mathrm{mrrm}]$, which could be attributed to the decreasing stereoselectivity of the catalyst with an increasing the amount of hydrogen. The decrease in the isotacticity can also be attributed to the decreasing molecular weights of these polymers with increasing hydrogen, which leads to loss in the active site control over the chain propagation.

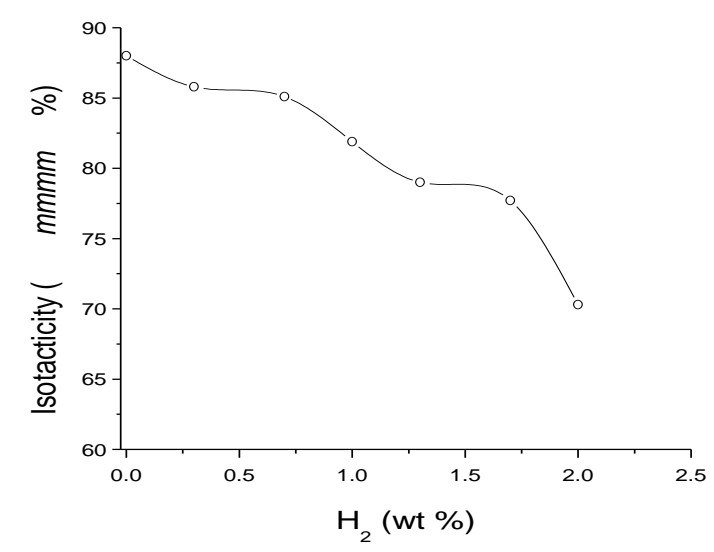

Figure 2: Dependence of the isotacticity of polypropylenes on the hydrogen content for $\mathrm{EI}(4 \mathrm{H})$ catalyst. 


\section{Fractionation and Characterization of Polypropylenes}

TREF analyses of three different isotactic polypropylenes D9, D8 and D7 produced using EI $(4 \mathrm{H})$ metallocene catalyst were carried out to determine the differences in their characteristics. Table 2 summarizes the TREF fractionation data of polypropylene samples D9, D8 and D7. Their TREF curves are shown in Figure 3.

Table 2: TREF fractionation data of the TREF fractions of samples D9, D8 and D7

\begin{tabular}{cccccc}
\hline Runs & Fraction $\left(\mathrm{T}_{\mathrm{e}}{ }^{\circ} \mathrm{C}\right)$ & $\mathrm{Wt}(\mathrm{mg})$ & $\mathrm{Wi} \%$ & $\Sigma \mathrm{Wi} \%$ & $\mathrm{Wi} \% / \Delta \mathrm{T}$ \\
\hline \multirow{5}{*}{ D9 } & 25 & 184 & 6.1 & 6.1 & - \\
& 60 & 226 & 7.5 & 13.6 & 0.4 \\
& 80 & 788 & 26.3 & 39.9 & 1.3 \\
& 90 & 1300 & 43.3 & 83.2 & 4.3 \\
& 100 & 337 & 11.2 & 94.4 & 1.1 \\
& 110 & 85 & 2.7 & 97.1 & 0.3 \\
& 130 & 89 & 2.9 & 100.0 & 0.2 \\
\hline \multirow{5}{*}{ D8 } & 25 & 520 & 16.8 & 16.8 & - \\
& 60 & 448 & 14.5 & 31.3 & 0.4 \\
& 80 & 952 & 30.8 & 62.1 & 1.5 \\
& 90 & 676 & 21.9 & 84.0 & 2.2 \\
& 100 & 373 & 12.1 & 96.1 & 1.2 \\
& 110 & 61 & 2.0 & 98.1 & 0.2 \\
D7 & 130 & 60 & 1.9 & 100.0 & 0.1 \\
\hline 25 & 650 & 21.7 & 21.7 & - \\
& 60 & 560 & 18.7 & 40.4 & 0.5 \\
& 80 & 1160 & 38.6 & 79.0 & 1.9 \\
& 90 & 370 & 12.3 & 91.3 & 1.2 \\
& 100 & 123 & 4.1 & 95.4 & 0.4 \\
\hline
\end{tabular}

Table 2 and the curves of the $\mathrm{W}_{\mathrm{i}} \% / \Delta \mathrm{T}$ versus the fractionation temperature in Figure 3(A) show that there is an increase in $\mathrm{W}_{\mathrm{i}} \% / \Delta \mathrm{T}$ for the first three fractions collected after the $25^{\circ} \mathrm{C}$ fraction, and then a decrease for the last three fractions. The curves of D9, D8 and D7 polymers in Figure 3(A) have different distributions and shapes. On the other hands, Figure 3(B) illustrates the accumulative weight fraction percentage versus the fractionation temperatures of the three polymers D9, D8 and D7. It is clear that the curves are considerably different, which is due to the differences in the molecular weight, tacticity and molecular weight distribution of the original polymers. The weight fraction as a function of elution temperature is shown in Figure 4. The superimposed graphs provide a clear and easy way to compare the samples. It is clear from the graph that the three samples have different weight fraction distributions. It is also observed that more than $50 \mathrm{wt} \%$ of the original material for all samples eluted at the 80 and $90{ }^{\circ} \mathrm{C}$ fractions. 

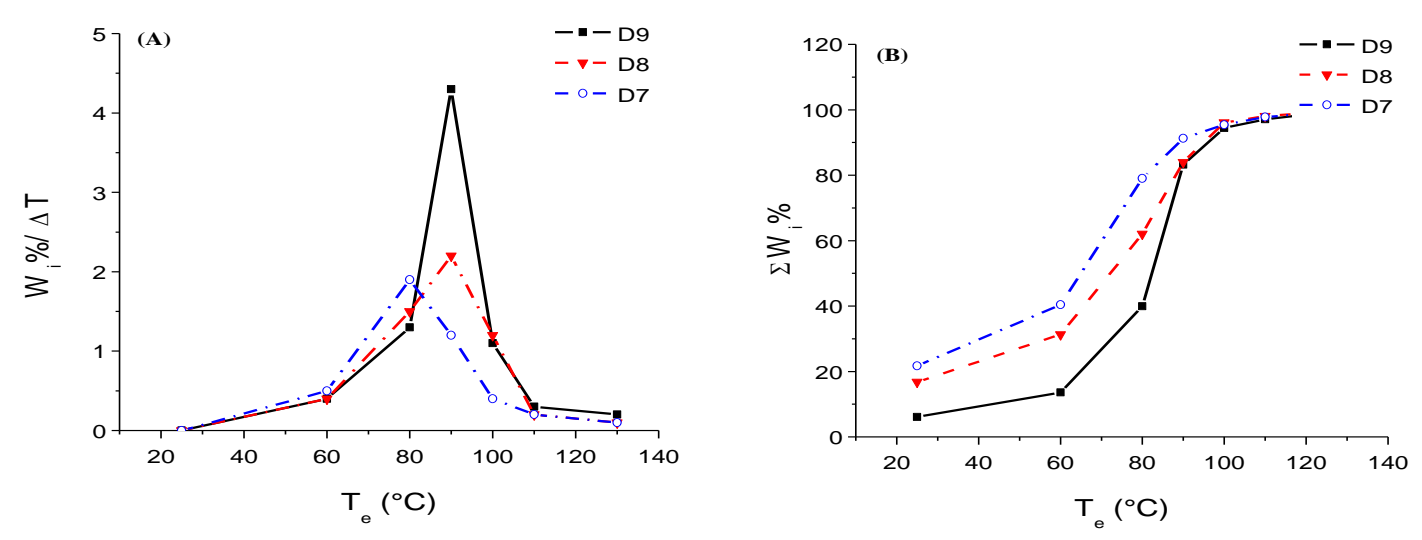

Figure 3: TREF curves of samples D9, D8 and D7: (A) Wi\%/DT and (B) $\Sigma W i \%$.

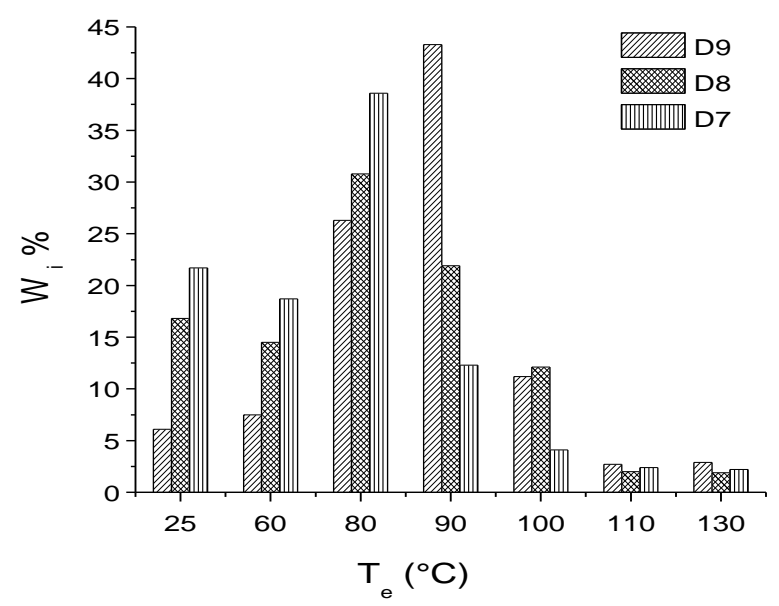

Figure 4: Distribution of weight percentage of D9, D8 and D7 fractions vs. elution temperature $\left({ }^{\circ} \mathrm{C}\right)$.

The characterization results of the fractions for D9, D8 and D7 polymers are summarized in Table 3. Other researchers have clearly demonstrated that for polypropylene samples the TREF profiles qualitatively reflect the distribution of isotacticity, with isotacticity increasing almost linearly with the elution temperature (Kioka et al., 1994; Viville et al., 2001; Soares \& Hamielec, 1996).

Table 3 shows that D9, D8 and D7 polymers are composed of fractions with different tacticities, ranging from rather atactic (elution temperature $25^{\circ} \mathrm{C}$ ) to highly isotactic (elution temperature $100{ }^{\circ} \mathrm{C}$ ). An interesting outcome of this analysis is that the isotacticity of D9, D8 fractions are systematically slightly greater than those of D7 fractions, at a given elution temperature. This is spite of the tacticity of the D9, D8 parent polymers being higher than that of the D7 parent polymer (Table 1).

The degree of crystallinity of TREF fractions of samples D8 and D7 increases with elution temperature for the first four fractions and then starts to decrease for the last two fractions, while it only increases for the first three TREF fractions of sample D9 and then starts to decrease as showed in Table 3 and Figure 5. 
Table 3: GPC, ${ }^{13} \mathrm{C}$ NMR and DSC Results of Fractionation Data of Samples D9, D8 and D7

\begin{tabular}{ccccccccc}
\hline Runs & $\begin{array}{c}\text { Fraction } \\
\left(\mathrm{T}_{\mathrm{e}}{ }^{\circ} \mathrm{C}\right)\end{array}$ & $\mathrm{M}_{\mathrm{w}}$ & $\mathrm{PD}$ & $m m m m \%$ & $\begin{array}{c}\mathrm{T}_{\mathrm{m}} \\
\left({ }^{\circ} \mathrm{C}\right)\end{array}$ & $\begin{array}{c}\mathrm{T}_{\mathrm{c}} \\
\left({ }^{\circ} \mathrm{C}\right)\end{array}$ & $\begin{array}{c}\Delta \mathrm{H}_{\mathrm{m}} \\
(\mathrm{J} / \mathrm{g})\end{array}$ & $\begin{array}{c}\mathrm{X}_{\mathrm{c}} \\
(\%)\end{array}$ \\
\hline \multirow{5}{*}{ D9 } & 25 & 1557 & 1.6 & 57.0 & - & - & - & - \\
& 60 & 7438 & 1.6 & 81.0 & 121.1 & 91.6 & 53.6 & 25.7 \\
& 80 & 20634 & 1.8 & 83.0 & 131.7 & 100.5 & 76.7 & 36.7 \\
& 90 & 28875 & 1.6 & 87.0 & 137.4 & 104.6 & 79.9 & 38.2 \\
& 100 & 26801 & 1.6 & 89.0 & 136.5 & 104.8 & 73.6 & 35.2 \\
& 110 & 28481 & 1.6 & 86.0 & 136.4 & 98.1 & 68.8 & 32.9 \\
& 130 & 22195 & 1.5 & - & 132.2 & 95.8 & 60.3 & 28.9 \\
\hline \multirow{5}{*}{ D8 } & 25 & 1420 & 1.4 & 56.0 & - & - & - & - \\
& 60 & 3463 & 1.4 & 78.0 & 122.9 & 91.8 & 78.6 & 37.6 \\
& 80 & 7042 & 1.5 & 84.0 & 135.7 & 104.1 & 85.0 & 40.7 \\
& 90 & 9664 & 1.6 & 89.0 & 140.0 & 107.3 & 89.9 & 43.0 \\
& 100 & 9777 & 1.6 & 91.0 & 141.0 & 109.2 & 95.8 & 45.8 \\
& 110 & 10645 & 1.3 & 87.0 & 139.5 & 101.8 & 86.4 & 41.3 \\
& 130 & 6930 & 1.2 & - & 133.7 & 98.5 & 81.9 & 39.2 \\
\hline \multirow{5}{*}{ D7 } & 25 & 1303 & 1.4 & 56.0 & - & - & - & - \\
& 60 & 3240 & 1.3 & 77.0 & 125.1 & 96.3 & 77.4 & 37.0 \\
& 80 & 6261 & 1.5 & 82.0 & 137.2 & 104.9 & 79.1 & 37.9 \\
& 90 & 8478 & 1.5 & 87.0 & 141.4 & 108.9 & 90.5 & 43.3 \\
& 100 & 8307 & 1.4 & 90.0 & 142.2 & 109.0 & 92.6 & 44.3 \\
& 110 & 7736 & 1.3 & 85.0 & 140.1 & 102.5 & 84.7 & 40.5 \\
& 130 & 5901 & 1.2 & - & 135.6 & 98.7 & 82.2 & 39.3 \\
\hline
\end{tabular}

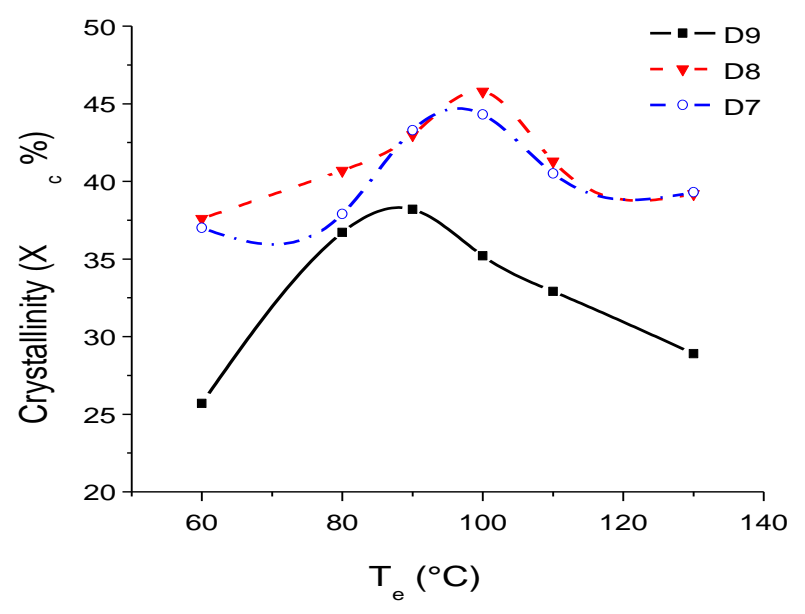

Figure 5: Degree of crystallinity of TREF fractions of samples D9, D8 and D7 versus TREF elution temperature.

The reason for the decreasing crystallinity of the last fractions is due to the significant decrease in the molecular weight and tacticity of these fractions, as evident in Table 3. Moreover, Figure 6 shows typical WAXD patterns of the $\alpha$ form of selected TREF fractions of sample D9, in which three strong equatorial peak of (110), (040) and (130) appear at $2 \theta=14.10^{\circ}, 16.90^{\circ}$ and $18.58^{\circ}$. 


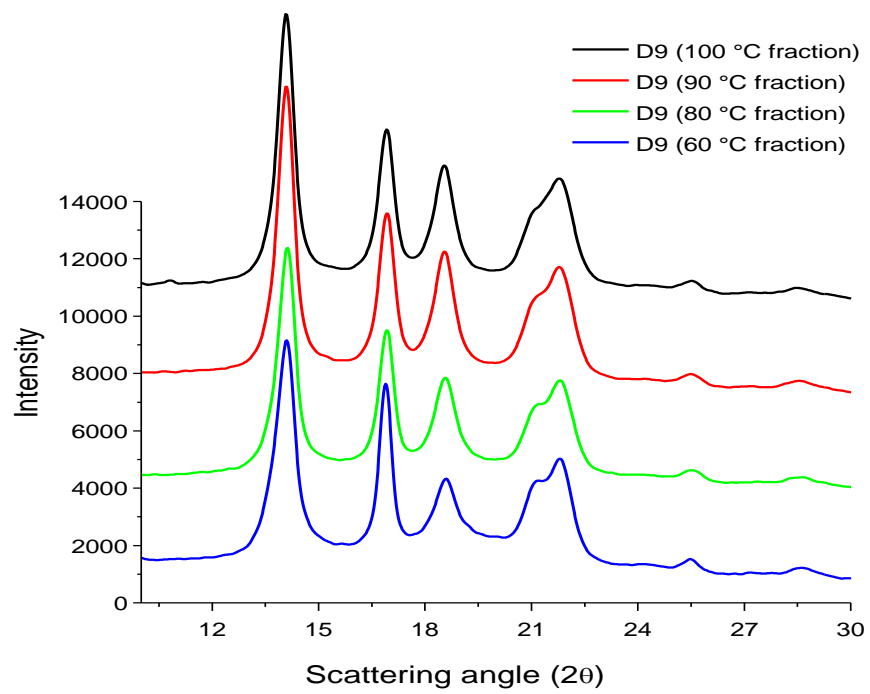

Figure 6: Typical X-ray diffraction pattern of various TREF fractions of sample D9.

\section{Conclusion}

By using metallocene catalyst system it was possible to produce polymer samples of a wide variation in tacticity and molecular weight. The addition of molecular hydrogen to propylene polymerizations catalyzed using the $\mathrm{EI}(4 \mathrm{H})$ catalyst system resulted in a reduction in polymer molecular weight and isotacticity of the polypropylenes. Furthermore, Preparative TREF allowed us to produce polymer fractions of well-defined structure in terms of molecular weight and distribution, as well as tacticity. A major increase in the degree of crystallinity of the polypropylene fractions was also noticed upon increasing the elution temperature. Moreover, X-ray diffraction showed only one crystal structure ( $\alpha$ phase) for the crystalline fractions of the polypropylene samples.

\section{Acknowledgements}

This work is based on the research supported by the National Research Foundation (Reference: KIC150312115016, UID: 97137).

\section{References}

Auriemma, F., De Rosa, C., Boscato, T. \& Corradini, P. 2001. The oriented $\gamma$ form of isotactic polypropylene. Macromolecules. 34(14): 4815-4826.

Busico, V., Cipullo, R., Caporaso, L., Angelini, G. \& Segre, A. L. 1998. C2- symmetric ansa-metallocene catalysts for propene polymerization: Stereoselectivity and enantioselectivity, Journal of Molecular Catalysis A: Chemical. 128(1-3): 53-64.

Carvill, A., Tritto, I., Locatelli, P. \& Sacchi, M. C. 1997. Polymer microstructure as a probe into hydrogen activation effect in ansa-zirconocene / methylaluminoxane catalyzed propene polymerizations. Macromolecules. 30(23): 7056-7062.

Deukkil, P., Kim, I., Yang, H., Soo, S., Kim, B. \& Chang, H. 2005. Isothermal crystallization behavior of metallocene-catalyzed isotactic polypropylene. Journal of Applied Polymer Science. 95(2): 231-237.

Kioka, M., Makio, H., Mizuno, A. \& Kashiwa, N. 1994. Tacticity distribution of polypropylene prepared by $\mathrm{MgCl}_{2}$-supported titanium catalyst. Polymer. 35(3): 580-583. 
Lin, S. \& Waymouth, M. 1999. Regioirregular propene insertion in polypropenes synthesized with unbridged bis(2-aryl)indenyl zirconium dichloride catalysts: Implications on activity. Macromolecules. 32(25): 8283-8290.

Lin, S., Kravchenko, R. \& Waymouth, R. M. 2000. Ethylene and hydrogen effects in propylene polymerization with 2-arylindenyl zirconocenes. Journal of Molecular Catalysis A: Chemical. 158(1): 423-427.

Moscardi, G., Piemontesi, F. \& Resconi, L. 1999. Propene polymerization with the isospecific, highly regiospecific rac-Me2C(3-t-Bu-1-Ind)2ZrCl2/MAO catalyst. 1. Influence of hydrogen on initiation and propagation: Experimental detection and theoretical investigation of 2,1 propene insertion into the $\mathrm{Zr}-\mathrm{H}$ bond. Organometallics. 18(25): 5264-5275.

Soares, J. B. \& Hamielec, A. E. 1996. Kinetics of propylene polymerization with a non-supported heterogeneous Ziegler-Natta catalyst - effect of hydrogen on rate of polymerization, stereoregularity, and molecular weight distribution. Polymer. 37(20): 4607-4614.

Soga, K. \& Shiono, T. 1997. Ziegler-Natta catalysts for olefin polymerizations. Progress in Polymer Science. 22(7): 1503-1546.

Tsutsui, T., Kashiwa, N. \& Mizuno, A. 1990. Effect of hydrogen on propene polymerization with ethylenebis(1indenyl)zirconium dichloride and methylalumoxane catalyst system. Macromolecular Rapid Communications. 11(11): 565-570.

Viville, P., Daoust, D., Jonas, A. M., Nysten, B., Legras, R., Dupire, M., Michel, J. \& Debrasb, G. 2001. Characterization of the molecular structure of two highly isotactic polypropylenes. Polymer. 42(5): 19531967.

Xu, J., Feng, L., Yang, S., Yang, Y. \& Kong, X. 1998. Temperature rising elution fractionation of polypropylene produced by heterogeneous Ziegler Natta catalyst. European Polymer Journal. 34(3-4): 431-434. 